\title{
Methoxychlor Induces CYP2C11 to Convert Itself into Hormonally Active Metabolites
}

\author{
Eriko Mikamo, Shingo Harada, Jun-ichi Nishikawa, and Tsutomu Nishihara* \\ Laboratory of Environmental Biochemistry, Graduate School of Pharmaceutical Sciences, Osaka University, 1-6 Yamada-oka, Suita, \\ Osaka 565-0871, Japan
}

(Received February 3, 2003; Accepted February 8, 2003)

\begin{abstract}
Methoxychlor (MXC), a chlorinated hydrocarbon pesticide, adversely affects the hormonal system in animals by producing metabolites with estrogenic activity. When methoxychlor was administrated to male rats for 7 days, CYP2C11 as well as its 2B1/2 and 3A1 was induced in the liver. In addition, an in vitro study using recombinant CYPs and HPLC analysis showed that only the CYP2C11 subfamily converted MXC into hormonally active metabolites, such as demethylated derivatives, not $2 \mathrm{~B} 1$ and $3 \mathrm{~A} 1$. These findings suggest that MXC accelerates the conversion of itself into hormonally active metabolites, leading to disruption of the endocrine system.
\end{abstract}

Key words — CYP2C11, methoxychlor, endocrine disruptor, hormonally active metabolite

\section{INTRODUCTION}

Methoxychlor [MXC; 1,1,1-trichloro-2,2-bis(4methoxyphenyl)ethane], a chlorinated hydrocarbon pesticide, is substituted for banned dichlorodiphenyltrichloroethane (DDT). Despite the relatively low toxicity in the acute phase $\left(\mathrm{LD}_{50}\right.$ : approximately $6 \mathrm{~g} /$ $\mathrm{kg}$ in rodent), it elicits reproductive toxicity in animals: Effect such as accelerated female pubertal development, delayed male pubertal development and reduced testosterone biosynthesis in male leydig cells were found in rodents. ${ }^{1-5)}$ Many studies have reported that phenolic metabolites of MXC are produced by hepatic cytochrome $\mathrm{P} 450$ monooxygenases (CYP), and MXC metabolites, mono- and bisdemethylated derivatives, show estrogenic and antiandrogenic activities by interacting with androgen

\footnotetext{
*To whom correspondence should be addressed: Laboratory of Environmental Biochemistry, Graduate School of Pharmaceutical Sciences, Osaka University, 1-6 Yamada-oka, Suita, Osaka 565-0871, Japan. Tel.: +81-6-6879-8240; Fax: +81-66879-8244; E-mail: nisihara@phs.osaka-u.ac.jp
}

and estrogen receptor, whereas the pure parent compound has no hormonal activities in vitro. ${ }^{6-10)}$ The tri-hydroxylated metabolite of MXC has only weak estrogenic activity, but not anti-androgenic activity. ${ }^{11)}$ In this paper, we investigated the association between the profile of CYP species induction and hormonally active metabolites of MXC. Administration of MXC induced CYP2C11, in addition to the 2B1/2 and $3 \mathrm{~A} 1$ in the rat liver, and only CYP2C11 subfamily could convert MXC into hormonally active metabolites, such as mono- and bis-demethylated derivatives, leading to disruption of the hormone balance.

\section{MATERIAL AND METHODS}

Chemicals - Methoxychlor (95\%) was purchased from Sigma chemical Co. (St. Louis, MO, U.S.A.). Insect cell microsomes expressing rat CYP2C11 were obtained from Daiichi Chemical Co. (Tokyo, Japan). Other reagents and solvents, unless otherwise cited, were from Nacalai Tesque, Inc. (Kyoto, Japan).

Animals Treatment and RT-PCR Analysis Male Sprague-Dawley rats (Crj:CD), 6 weeks of age, were purchased from Charles River Laboratories Japan, Inc. (Shiga, Japan) and acclimated for a week. Animals were gavaged with MXC $(50,100$, or $250 \mathrm{mg} / \mathrm{kg} / \mathrm{day}$ ) in corn oil or vehicle alone for 7 days. On the day following the final dose, animals were killed by decapitation and the organ weight was checked. Approximately $0.1 \mathrm{~g}$ frozen liver was homogenized and RNA was isolated using Trizol reagent (Invitrogen, Tokyo, Japan). For the quantitative RT-PCR assay, we performed competitive RTPCR using TAKARA RNA LA PCR ${ }^{\mathrm{TM}}$ (AMV) Kit Ver. 1.1 and Rat Cytochrome P450 Competitive RTPCR Set (TaKaRa, Ootsu, Japan) according to the manufacturer's instructions. Then, aliquots of PCR 
Table 1. Effect of Methoxychlor on Body and Organ Weight

\begin{tabular}{lccccccc}
\hline \hline Dose & Number & Body & Testes & Epididymides & $\begin{array}{c}\text { Seminal vesicles } \\
(\mathrm{g})\end{array}$ & $(\mathrm{g})$ & \multicolumn{2}{c}{$\begin{array}{c}\text { Prostate }(\mathrm{mg}) \\
\text { Dorso-lateral }\end{array}$} \\
\hline Control & 6 & $321 \pm 4.4$ & $2.83 \pm 0.08$ & $0.48 \pm 0.03$ & $655.1 \pm 29.4$ & $328.9 \pm 15.1$ & $184.5 \pm 10.6$ \\
$50 \mathrm{mg} / \mathrm{kg}$ & 5 & $302 \pm 6.6$ & $2.65 \pm 0.09$ & $0.51 \pm 0.01$ & $591.7 \pm 25.0$ & $294.9 \pm 25.6$ & $151.7 \pm 14.9$ \\
$100 \mathrm{mg} / \mathrm{kg}$ & 6 & $285 \pm 3.8^{*}$ & $2.62 \pm 0.17$ & $0.48 \pm 0.02$ & $521.1 \pm 41.6$ & $278.1 \pm 32.0$ & $143.1 \pm 9.0$ \\
$250 \mathrm{mg} / \mathrm{kg}$ & 6 & $271 \pm 7.5^{*}$ & $2.46 \pm 0.23$ & $0.42 \pm 0.04$ & $238.6 \pm 48.0^{*}$ & $205.5 \pm 27.6^{*}$ & $78.5 \pm 16.6^{*}$ \\
\hline
\end{tabular}

Values represent means \pm S.E. $* p<0.01$, Significantly different from the control group; Dunnett's multiple comparison.

products were electrophoresed and the amount of each separated band was quantified densitometrically using the public domain NIH Image Program version 1.60 .

Analysis of Methoxychlor Metabolites by HPLC and EI-MS - Incubation mixtures contained 10 pmol each CYP protein, $100 \mu \mathrm{M}$ MXC, $1.3 \mathrm{mM}$ $\mathrm{NADP}+, 3.3 \mathrm{mM}$ glucose-6-phosphate, $3.3 \mathrm{mM}$ $\mathrm{MgCl}_{2}$ and $0.4 \mathrm{U} / \mathrm{ml}$ glucose-6-phosphate dehydrogenase in $100 \mathrm{mM}$ potassium phosphate buffer $(\mathrm{pH} 7.4)$ to give a final volume of $0.5 \mathrm{ml}$. After the 0 or 60 min incubation period, the reaction was terminated by the addition of $200 \mu \mathrm{l}$ ice-cold ethyl acetate and the aqueous phase was extracted with $200 \mu$ l ethyl acetate. Metabolites were then analyzed by UV-HPLC and EI-MS.

\section{RESULTS AND DISCUSSION}

MXC has been found to elicit reproductive toxicity in both male and female rats. ${ }^{4,5)}$ First, we administered MXC to male rats for 7 days and investigated the effects, especially in the reproductive organs. MXC caused atrophy of reproductive organs, such as seminal vesicles and prostates, compared to the control group (Table 1). These results were consistent with the previous report. ${ }^{1)}$ Although the final total body weight was decreased, daily food consumption was not significantly different and the clinical tests showed no signs of general systematic toxicity (data not shown). The atrophy of reproductive organs can be characterized as estrogenic and antiandrogenic action of MXC.

The phenolic metabolites of MXC (Fig. 1), mono- and bis- demethylated derivatives, have estrogenic and anti-androgenic activity, despite the parent compounds not having any hormonal activity. ${ }^{6-10)}$ Although it has been reported that MXC induces CYP2B and $3 \mathrm{~A},{ }^{11)}$ it remains to be elucidated whether these enzymes produce such phenolic me-

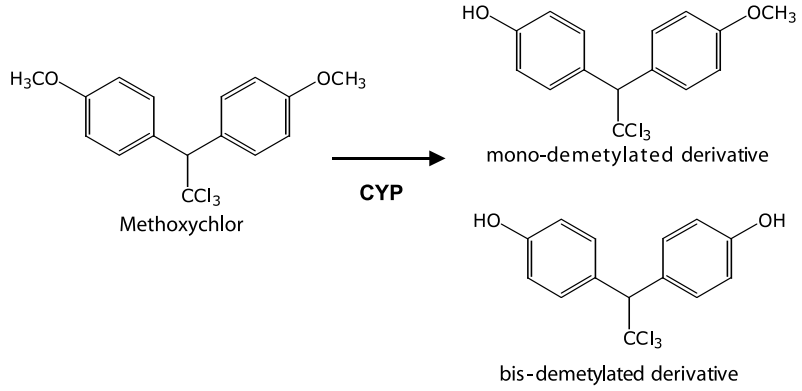

Fig. 1. Chemical Structures of Methoxychlor and Its Phenolic Metabolites

tabolites. Thus, we analyzed the expression pattern of CYP species in response to administration of MXC. After 7 consecutive treatments, the liver was removed and RNA was isolated for competitive RTPCR assay.

As shown in Fig. 2, CYP2C11 mRNA increased in a dose-dependent manner as well as CYP2B1/2 and 3A1. On the other hand, CYP1A1 mRNA was not detected, and CYP1A2 and CYP4A1 mRNA levels were not changed in this condition (data not shown). The CYP2C11 mRNA level at the highest dose group was almost equal to that in the control group. Our findings suggested that accumulated metabolites of MXC interfered with the induction of CYP2C11 mRNA. Androgens increase the CYP2C11 level and estrogens suppress the expression level, ${ }^{12)}$ and metabolites of MXC show antiandrogenic and estrogenic activity.

After the incubation of MXC in a reconstituted system containing each CYP species, the reaction mixtures were analyzed by UV-HPLC. As a result of HPLC analysis, two additional peaks, M1 and M2, were eluted at 14 and $18 \mathrm{~min}$ from the reaction mixture with CYP2C11 (Fig. 3A). However, no novel peaks were observed in chromatograms with CYP2B1 and CYP3A1 (data not shown). Additionally, when MXC was incubated with normal rat liver microsomes, major metabolites corresponded with 

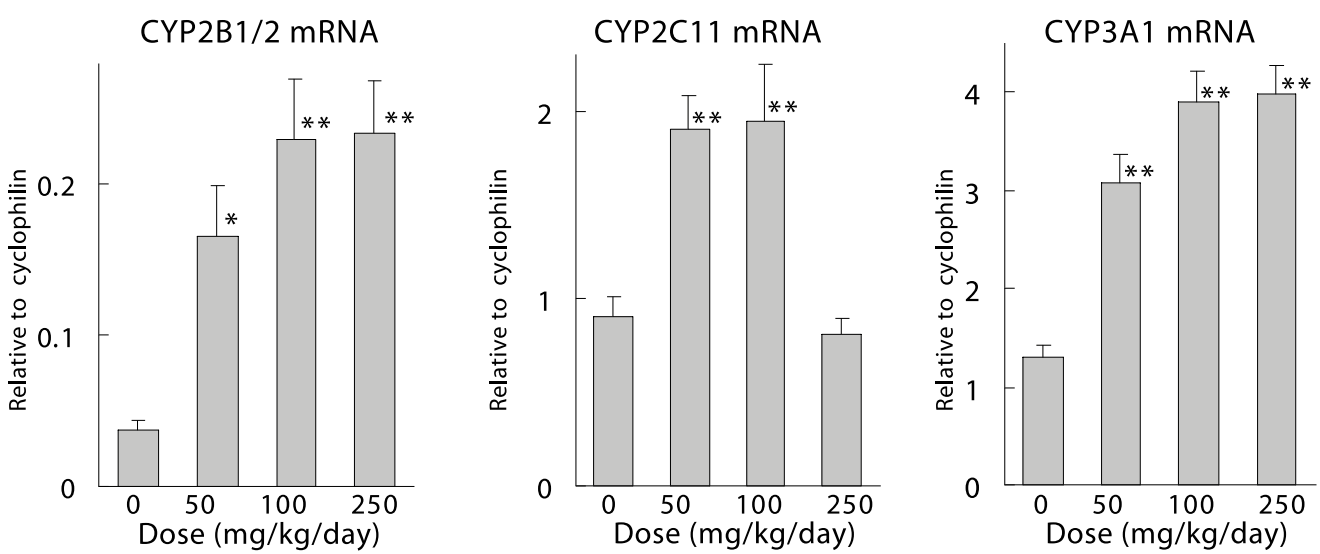

Fig. 2. Effect of Methoxychlor on Expression Pattern of CYP2B1/2, CYP2C11 and CYP3A1 mRNA in Rat Liver

Rats were orally administered 50,100 or $250 \mathrm{mg} / \mathrm{kg} / \mathrm{day}$ methoxychlor in corn oil or vehicle alone for 7 days. The amount of CYP mRNA were calculated from densitometrically and normalized by cyclophilin. Values represent means \pm S.E. of 5-6 rats (separated RNA isolation and RT-PCR reactions per rat) $* p<0.05,{ }^{* *} p<0.01$ Dunnett's multiple comparison.

A

mAbs

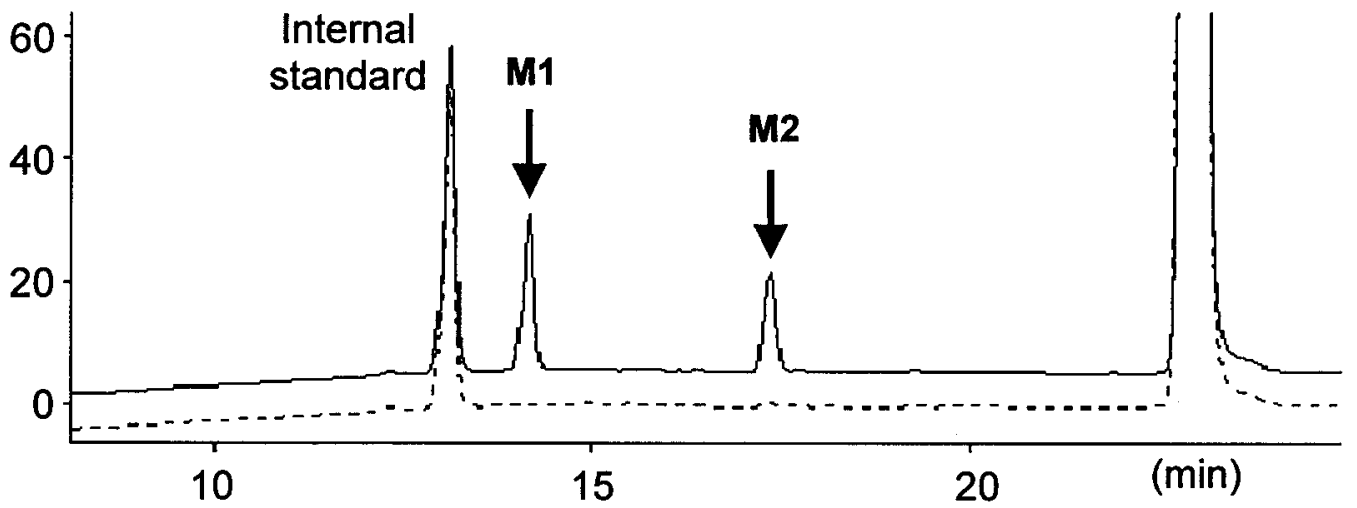

B
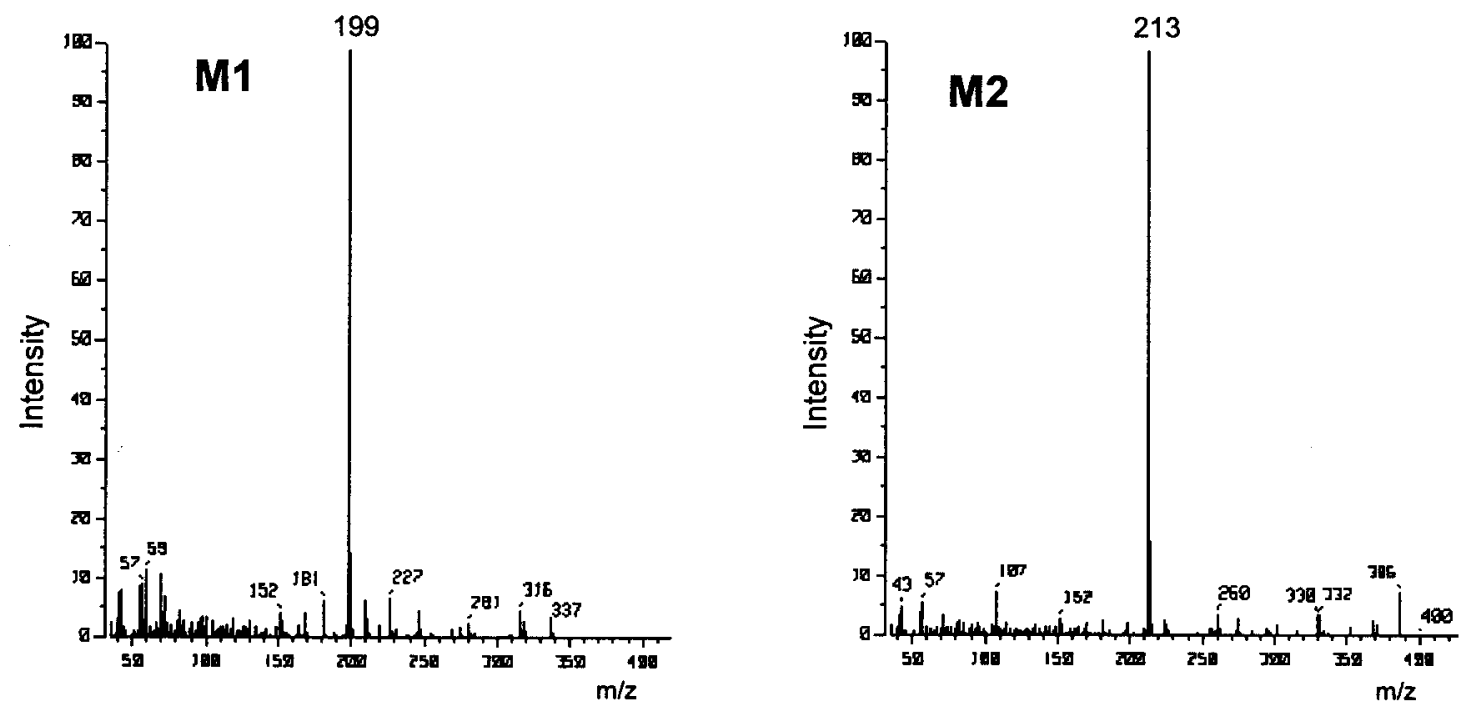

Fig. 3. Identification of Methoxychlor Metabolites Produced by CYP2C11

A: Methoxychlor metabolites were analyzed by UV-HPLC. The HPLC (Shimadzu: LC-10A) equipped with UV detector (SPD-10A) was operated as follows; column, Inertsil ODS-3 $(250 \times 4.6 \mathrm{~mm}$ i.d. $)$ with guard column $(5 \times 4.6 \mathrm{~mm}$ i.d.); mobile phase, methanol-water gradient; $\mathrm{UV}$ detection, $240 \mathrm{~nm}$; column temperature, $45^{\circ} \mathrm{C}$. (- - -), before; (-), after 60 min incubation; M1 and M2, methoxychlor metabolites. B: Mass spectra of methoxychlor metabolites. The MS spectrometer (JEOL: JMS-AX505W) was operated as follows: ionization mode, electron impact; ion source temperature, $230^{\circ} \mathrm{C}$; electron energy, $70 \mathrm{eV}$. 
M1 and M2 produced by the CYP2C11 (data not shown). These two metabolites were isolated by TLC and then applied to EI-MS. The base peak of MXC was detected at $\mathrm{m} / \mathrm{z} 227$ consistent with loss of trichlorinated methyl group (data not shown). Mass spectra showed that the ions with the highest abundant of each metabolite were at $\mathrm{m} / \mathrm{z} 199$ and 213 (Fig. 3B). Therefore, M1 and M2 were identified as bis- and mono-demethylated metabolite, respectively.

The expression of the CYP2C11 is sexually predominant in male rats, but it has been demonstrated that dexamethasone, lindane, oxandrolone and phenytoin also induce CYP2C11 in female rats, ${ }^{12,13)}$ suggesting that MXCis converted into phenolic metabolites through CYP2C11 in both males and females.

These findings suggest that MXC is converted into estrogenic and anti-androgenic phenolic metabolites by the CYP2C11, not by the CYP2B and 3A1, which is induced by MXC itself. Accordingly, MXC accelerates the conversion of itself into hormonally active metabolites, leading to disturbance of the endocrine system.

Acknowledgements This work was supported in part by grants from the Japanese Ministries of Education, Culture, Sports, Science and Technology, and of Health, Labour and Wellfare.

\section{REFERENCES}

1) Linder, R. E., Strader, L. F., Slott, V. L. and Suarez, J. D. (1992) Endpoints of spermatotoxicity in the rat after short duration exposures to fourteen reproductive toxicants. Reprod. Toxicol., 66, 491505.

2) Cummings, A. M. (1997) Methoxychlor as a model for environmental estrogens. Crit. Rev. Toxicol., 27, 367-379.

3) Akingbemi, B. T., Ge, R. S., Klinefelter, G. R., Gunsalus, G. L. and Hardy, M. P. (2000) A metabolite of methoxychlor, 2,2-bis(p-hydroxyphenyl)-1,1, 1trichloroethane, reduces testosterone biosynthesis in rat leydig cells through suppression of steady-state messenger ribonucleic acid levels of the cholesterol side-chain cleavage enzyme. Biol. Reprod., 62, 571578.

4) Wade, M. G., Foster, W. G., Younglai, E. V., McMahon, A., Leingartner, K., Yagminas, A., Blakey, D., Fournier, M., Desaulniers, D. and
Hughes, C. L. (2002) Effects of subchronic exposure to a complex mixture of persistent contaminants in male rats: systemic, immune, and reproductive effects. Toxicol. Sci., 67, 131-143.

5) Chapin, R. E., Harris, M. W., Davis, B. J., Ward, S. M., Wilson, R. E., Mauney, M. A., Lockhart, A. C., Smialowicz, R. J., Moser, V. C., Burka, L. T. and Collins, B. J. (1997) The effects of perinatal/juvenile methoxychlor exposure on adult rat nervous, immune, and reproductive system function. Fundam. Appl. Toxicol., 40, 138-157.

6) Dehal, S. S. and Kupfer, D. (1994) Metabolism of the proestrogenic pesticide methoxychlor by hepatic P450 monooxygenases in rats and humans. Drug Metab. Dispos., 22, 937-945.

7) Bulger, W. H. and Kupfer, D. (1990) Studies on the formation of methoxychlor-protein adduct in rat and human liver microsomes. Is demethylation of methoxychlor essential for cytochrome P450 catalyzed covalent binding? Biochem. Pharmacol., 40, 937-945.

8) Gaido, K. W., Maness, S. C., Mcdonnell, D. P., Dehal, S. S. and Kupfer, D. (2000) Interaction of methoxychlor and related compounds with estrogen receptor $\mathrm{a}$ and $\mathrm{b}$, and androgen receptor: structureactivity studies. Mol. Pharmacol., 58, 852-858.

9) You, L., Casanova, M., Bartolucci, E. J., Fryczynski, M. W., Dorman, D. C., Everitt, J. I., Gaido, K. W., Ross, S. M. and Heck, Hd H. (2002) Combined effects of dietary phytoestrogen and synthetic endocrine-active compound on reproductive development in Sprague-Dawley rats: genistein and methoxychlor. Toxicol. Sci., 66, 91-104.

10) $\mathrm{Hu}, \mathrm{Y}$. and Kupfer, D. (2002) Enantioselective metabolism of the endocrine disruptor pesticide methoxychlor by human cytochromes p450 (p450s): major differences in selective enantiomer formation by various p450 isoforms. Drug Metab. Dispos., 30, 1329-1336.

11) Li, H. C. and Kupfer, D. (1998) Mechanism of induction of rat hepatic CYP2B and $3 \mathrm{~A}$ by the pesticide methoxychlor. J. Biochem. Mol. Toxicol., 12, 315-323.

12) Waskiewicz, M. J., Choudhuri, S., Vanderbeck, S. M., Zhang, X. J. and Thomas, P. E. (1995) Induction of "male-specific" cytochrome P450 isozymes in female rats by oxandrolone. Drug Metab. Dispos., 23, 1291-1296.

13) Oropeza-Hernandez, L. F., Sierra-Santoyo, A., Cebrian, M. E., Manno, M. and Albores, A. (2001) Ovariectomy modulates the response of some cytochrome P450 isozymes to lindane in the rat. Toxicol. Lett., 124, 91-99. 